\title{
Design of NEID, an extreme precision Doppler spectrograph for WIYN
}

\author{
C. Schwab ${ }^{a, b}$, A. Rakich ${ }^{c}$, Q. Gong ${ }^{d}$, S. Mahadevan ${ }^{e}$, S. P. Halverson ${ }^{e}$, A. Roy ${ }^{e}$, R. C. Terrien ${ }^{e}$, \\ P. M. Robertson ${ }^{e, f}$, F. R. Hearty ${ }^{e}$, E. I. Levi ${ }^{e}$, A. J. Monson ${ }^{e}$, J. T. Wright ${ }^{e}$, M. W. McElwain ${ }^{d}$, \\ C. F. Bender ${ }^{e}$, C. H. Blake ${ }^{g}$, J. Stürmer ${ }^{h}$, Y.V. Gurevich ${ }^{i}$, A. Chakraborty ${ }^{j}$, L. W. Ramsey ${ }^{e}$
}

${ }^{a}$ Department of Physics and Astronomy, Macquarie University, Australia;

${ }^{b}$ Australian Astronomical Observatory, Australia;

${ }^{c}$ Giant Magellan Telescope Organization Corp., USA;

${ }^{d}$ ExoPlanets and Stellar Astrophysics Laboratory, Goddard Space Flight Center, USA;

${ }^{e}$ Department of Astronomy \& Astrophysics, The Pennsylvania State University, USA;

${ }^{\mathrm{N}} \mathrm{ASA}$ Carl Sagan Fellow

${ }^{g}$ Department of Physics and Astronomy, University of Pennsylvania, USA;

${ }^{h}$ Department of Astronomy and Astrophysics, University of Chicago, USA;

iZAH-Landessternwarte Heidelberg, Germany;

${ }^{j}$ Physical Research Lab, Ahmedabad, India

\begin{abstract}
We have developed an optical design for a high resolution spectrograph in response to NASA's call for an extreme precision Doppler spectrometer (EPDS) for the WIYN telescope. Our instrument covers a wavelength range of 380 to $930 \mathrm{~nm}$ using a single detector and with a resolution of 100,000. To deliver the most stable spectrum, we avoid the use of an image slicer, in favor of a large (195 mm diameter) beam footprint on a 1x2 mosaic R4 Echelle grating. The optical design is based on a classic white pupil layout, with a single parabolic mirror that is used as the main and transfer collimator. Cross dispersion is provided by a single large PBM2Y glass prism. The refractive camera consists of only four rotationally symmetric lenses made from i-Line glasses, yet delivers very high image quality over the full spectral bandpass. We present the optical design of the main spectrograph bench and discuss the design trade-offs and expected performance.
\end{abstract}

Keywords: Echelle spectrograph, Doppler technique, radial velocities, exoplanets, fibre-fed spectrograph.

\section{INTRODUCTION}

Our team has responded to a call by NASA to design an extreme precision Doppler spectrometer for the $3.5 \mathrm{~m}$ WIYN telescope at Kitt Peak National Observatory in Arizona. The goal was to deliver an instrument concept that enables unprecedented radial velocity (RV) precision of better than $50 \mathrm{~cm} / \mathrm{s}$ in a single measurement and provides a credible path towards $10 \mathrm{~cm} / \mathrm{s}$. This level of precision is necessary to detect Earth-like planets in the Habitable Zone of nearby stars. Instruments with this level of sensitivity are critically needed to enable ground-based follow-up of planets discovered by NASA's TESS mission, which is scheduled to launch in 2017.

To achieve these science goals, the spectrograph must provide high resolution (R 100,000), utmost stability of the point spread function (PSF), wide wavelength coverage extending into the NIR region that contains important indicators of stellar activity, and high efficiency. To achieve the ultimate instrument stability, we opted to use a single fiber to couple the telescope to the spectrograph and to avoid the use of image slicers or reformatters, which exacerbate modal noise and have not been shown to deliver sub-m/s precision ${ }^{l}$. With a fiber aperture of 0.92 arcseconds on sky, the fully illuminated $1 \times 2$ mosaic R4 Echelle grating with a blaze angle of $76^{\circ}$ delivers the desired resolution $(\mathrm{R}=100,000)$. Our spectrograph is based on a white pupil relay (WPR) ${ }^{2,3}$, which is commonly used in state-of-the-art high resolution spectrometers. We use sub-apertures of a single monolithic parabolic white pupil mirror that provides unity magnification in the relay. We 
use a single camera to cover the full spectral range $(380-930 \mathrm{~nm})$ of the instrument (see Fig. 1). Since the spectral range is larger than an octave, we use a prism rather than a grating as the cross-disperser. The design is fully based on i-Line glasses, which deliver excellent transmission down into the UV. The use of a prism cross-disperser and the single camera design with very few optical elements produces a spectrograph with excellent throughput.

NEID was designed to fit into the existing vacuum tank design developed for the Habitable Zone Planet Finder (HPF), which delivers excellent thermal stability ${ }^{4}$. In this paper, we present the optical design of the spectrograph bench.

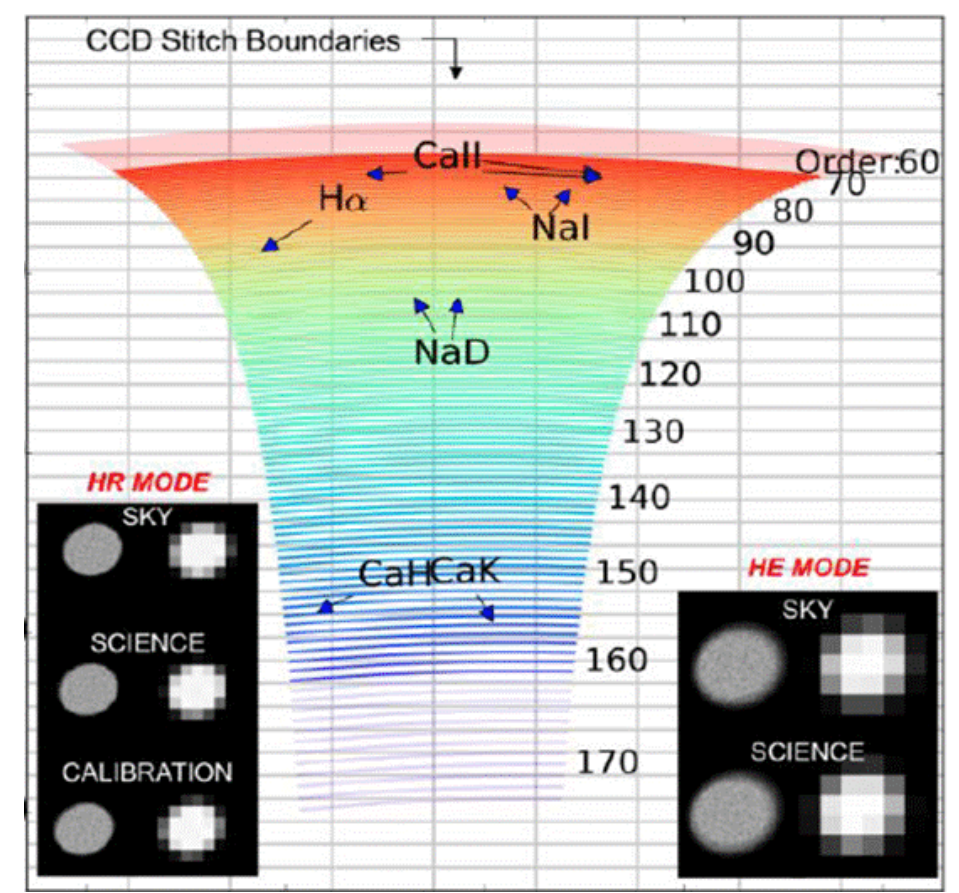

Fig. 1. Spectral format on the $9 \mathrm{k} x$ $9 \mathrm{k}$ e2v CCD $290-99$ detector. Order numbers are given on the right, and the grid indicates the CCD stich boundaries. Blue arrows show the locations of important stellar activity indicators. The small insets show the shape, relative location and sampling of our fibers in high resolution mode (HR; 62 micron fiber diameter with $\sim 5$ pixel sampling, as shown on the right side of the inset image) and high efficiency mode (HE; 100 micron fiber diameter, shown binned into 2x2 pixels in the right inset image).

\section{OPTICAL DESIGN}

The optical design of the spectrograph bench comprises four subsections: the fiber injection, the WPR, the cross disperser, and the camera optics.

\subsection{White pupil relay}

Fig. 2 shows the optical layout of the spectrograph. The white pupil relay is based on a monolithic parabolic mirror used in triple pass. The grating is slightly tilted $\left(\gamma=0.5^{\circ}\right)$ towards the optical axis of the primary mirror to separate the incident beam from the dispersed beam. In our design, the fiber input is between the grating and the flat fold mirror.

The primary mirror will be manufactured on a single Zerodur substrate with a diameter of $590 \mathrm{~mm}$ and a focal length of $1550 \mathrm{~mm}$. The substrate will be cut into a rectangular shape for ease of mounting and to allow for a low optical axis. Using a single mirror instead of two off-axis parabolas (OAPs) reduces the number of degrees of freedom in the optomechanics, leading to a more stable spectrograph. The relatively relaxed focal ratio (f/2.6) of this mirror in comparison to OAPs used in white pupil relays of other existing designs ${ }^{5,6}$ facilitates manufacturing and loosens the alignment tolerances. Since this mirror is used in triple pass, the efficiency of its coating is crucial for obtaining optimal throughput. Thus, we are considering both enhanced metallic mirror coatings and fully dielectric coatings in order to achieve the best possible reflectivity down to $380 \mathrm{~nm}$. 
The Echelle grating we will use is a R4 grating with 31.6 lines/mm from Newport/Richardson Grating Labs. It is a $1 \times 2$ mosaic with a total ruled area of $200 \times 800 \mathrm{~mm}$ on a single Zerodur substrate. We have requested Zerodur Class 0 Extreme to minimize sensitivity to thermal transients. Our grating will be replicated from Master MR263, the same master that is used for the grating of the ESPRESSO spectrograph. Our design yields a $195 \mathrm{~mm}$ diameter beam at the grating, which fills the projected aperture size of the grating. Since Newport does not offer enhanced silver coatings on substrates of this size, we will use an enhanced aluminum coating.

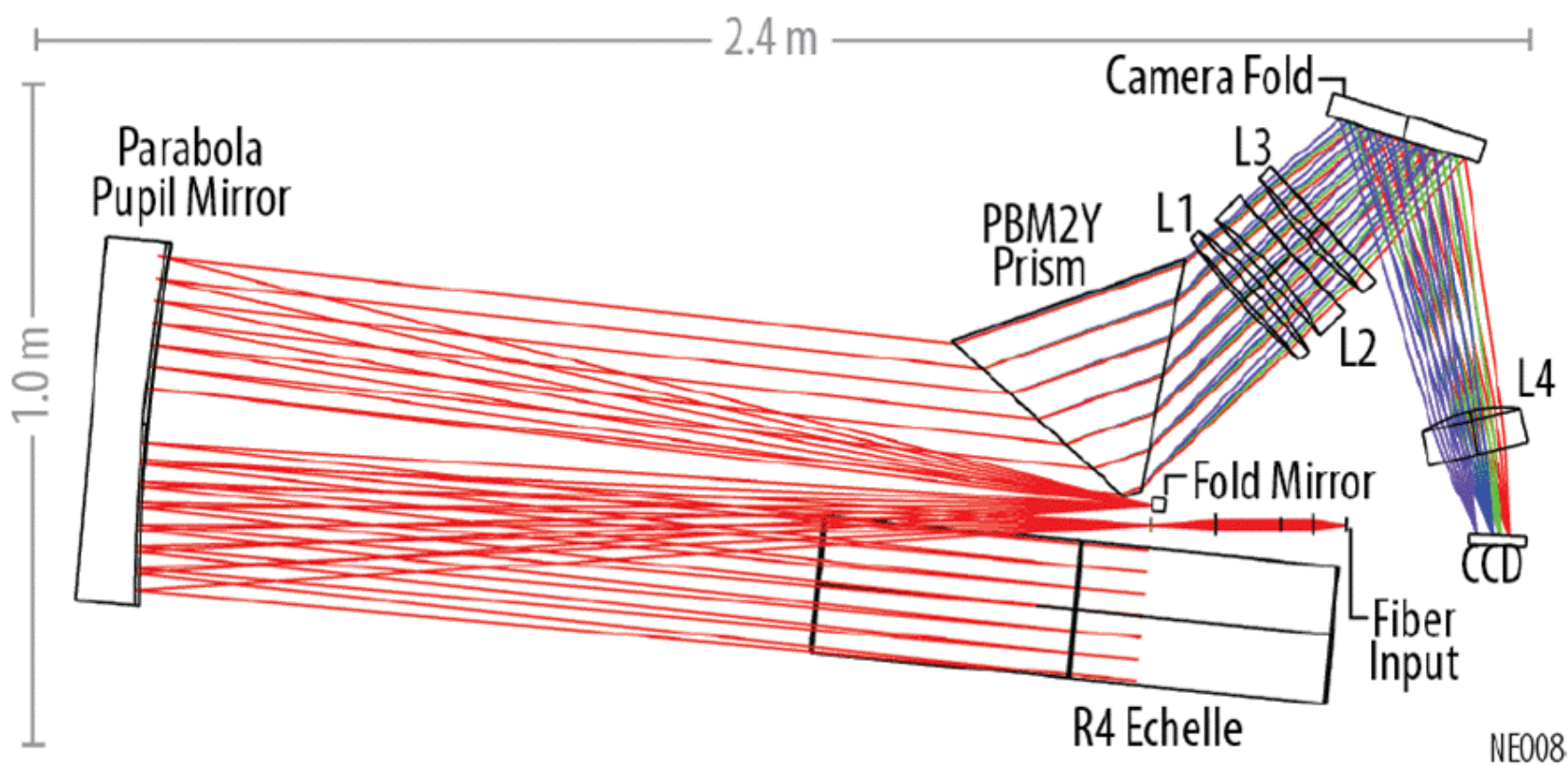

Fig. 2. Optical layout of NEID.

\subsection{Cross disperser}

Since the ratio between the longest and shortest wavelengths in the NEID spectral range is $>2$, using a grating cross disperser would result in overlap of the orders. A prism also provides higher throughput than a grating because the efficiency of gratings typically decreases towards the edges of their spectral range. Our white pupil relay provides unity magnification so we require a relatively small amount of cross dispersion. This, combined with our decision not to use image slicers, enables us to achieve the required cross dispersion with a single prism. Since our wavelength range reaches into the UV, we use extremely pure lithography glasses optimized for excellent transmission down to the i-Line $(365 \mathrm{~nm})$. The prism will be made from Ohara Corporation's PBM2Y type glass, which is a flint (equivalent to the common Schott F2) that has internal transmission $>99.5 \%$ per $\mathrm{cm}$ and an Abbe number $V_{D}=36.3$. This is the lowest Abbe number of all currently available i-Line glasses.

Ohara has agreed to melt rectangular blocks of PBM2Y of sufficient size to yield a monolithic prism. Due to the long lead time and associated schedule risk to the project, we will purchase two such blocks. The guaranteed refractive index homogeneity is better than $5 \mathrm{ppm}$, possibly requiring local retouching of the prism face(s) to achieve the desired transmitted wavefront error of $<\lambda / 4$. This technique has successfully been employed for the cross disperser prism of the LEVY spectrograph at the $2.4 \mathrm{~m}$ APF telescope ${ }^{7}$. The clear aperture of the prism is roughly $380(\mathrm{~W}) \times 250(\mathrm{H}) \mathrm{mm}$

We require a sufficiently large interorder spacing to guarantee clean extraction of adjacent orders. This is achieved by using a prism with a $60^{\circ}$ apex angle in minimum deviation. The incidence angle at the prism entrance face is $54^{\circ}$. This makes an efficient anti-reflection coating challenging; however, we are confident that an average reflectivity of less than $2.5 \%$ per surface can be achieved with specialized dielectric coatings. 


\subsection{Camera optics}

To image the full Echelle spectral format onto our detector, we use a refractive camera with a $250 \mathrm{~mm}$ entrance aperture. The design requirements were a focal length of $\sim 600 \mathrm{~mm}$ and a large field of view to illuminate our large $90 \times 90 \mathrm{~mm}$ CCD detector. We have chosen to optimize the camera optics to achieve minimal spot diameters for all orders between 380 and $750 \mathrm{~nm}$; this results in a slight roll-off in image quality at the ends of the long red orders between 750 and 930 $\mathrm{nm}$.

We optimized the system to achieve the smallest possible spot sizes in order to make the PSF as insensitive as possible to incomplete scrambling in the pupil plane. Fig. 3 demonstrates the image quality we achieve. In our prioritized spectral range $(380-750 \mathrm{~nm})$, the image quality approaches the diffraction limit, with $100 \%$ ensquared energy in a 10 micron pixel. Note that one resolution element in the center of the order corresponds to 50 microns or 5 pixels. The natural symmetry of the relay optics leads to an approximately symmetric aberration distribution about the center of each order, as can be seen in the spot diagrams.

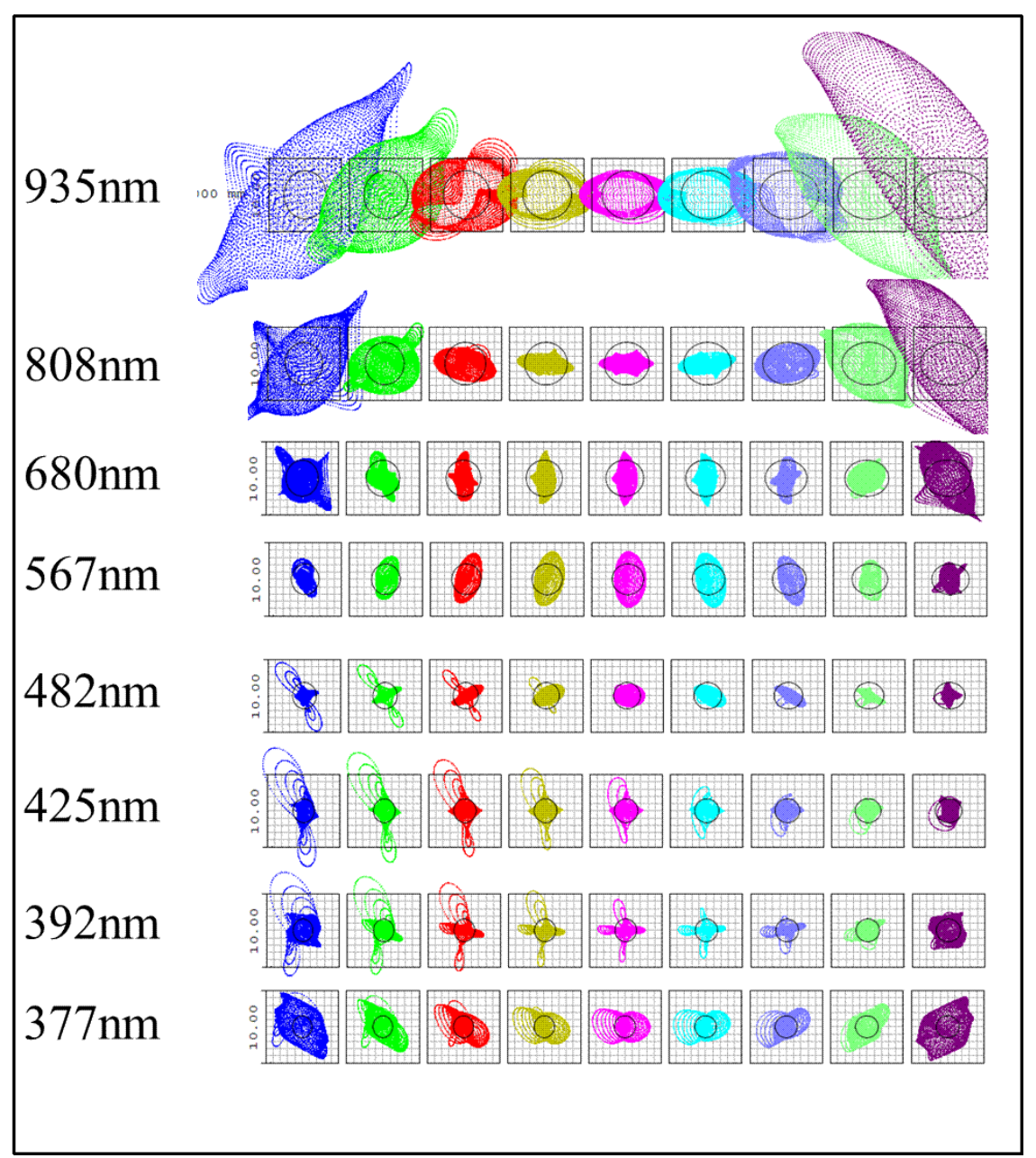

Fig. 3. Spot diagrams for the central (HR science) fiber in selected orders, showing nine wavelengths per order spread evenly across the free spectral range. Box sizes are 10 micron / 1 pixel. The small black circles indicate the local Airy disc diameters. 
The optical layout of the camera is shown in Fig. 4. The camera consists of four all-spherical lenses arranged in a front triplet group and a singlet near the detector. We kept a large back focal distance, which helps in avoiding ghosts from the back reflection off the detector surface. The detector is mounted at a relatively large tilt angle of $15.7^{\circ}$ in the crossdispersion direction to remove chromatic defocus. The camera is telecentric in the dispersion direction to make the spot positions insensitive to small focus variations. The large separation between the front triplet group and the singlet allows us to insert a flat fold mirror. The fold mirror is necessary due to the requirement that the optics fit into the space envelope of the optical bench and vacuum tank developed for the HPF spectrograph.

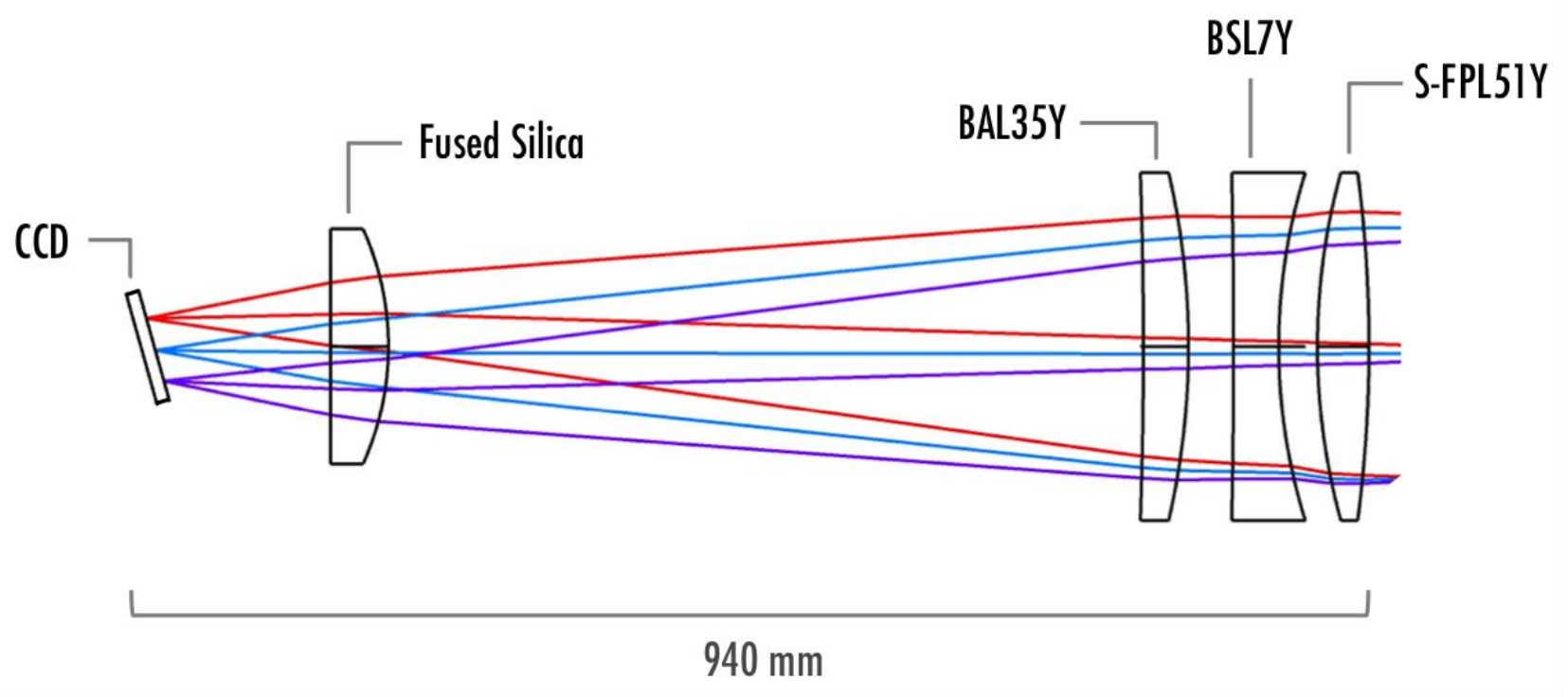

Fig. 4. Optical layout of the camera (shown without the fold). All surfaces are spherical. The front triplet group is concentric, while the last lens is tilted slightly to correct remaining aberrations. All glasses are from Ohara's i-Line catalogue and have excellent transmission into the UV. The last lens is made from fused silica to avoid extra dark counts on the CCD due to radioactive decay commonly associated with many glass types.

The white pupil relay we use has intrinsic cylindrical field curvature due to the Petzval sum of the mirrors. This is often removed by using a cylindrical lens. However, to act as an efficient field flattener, the cylindrical lens must be mounted very close to the detector, which leads to difficulties with avoiding ghosts and mounting the detector. We avoid the use of a cylindrical field flattener in favor of tilting and decentering the last lens in the cross-dispersion direction. This balances the remaining aberrations and removes the cylindrical field curvature, while providing a significantly larger back focal length $(137 \mathrm{~mm})$ than is possible with a cylindrical lens.

The camera lenses are made from Ohara Corporation's i-Line glasses, which deliver excellent throughput down into the UV. These glasses are available as custom blanks with diameters $>250 \mathrm{~mm}$ and excellent homogeneity. The singlet in front of the detector will be made from fused silica to avoid any additional dark signal due to radioactive decays in the lens material that is in close proximity to the detector.

For the detector, we use the CCD-290-99 device from e2v with $9216 \times 923110 \mu \mathrm{m}$ pixels, the same detector used for the ESPRESSO spectrograph at the VLT. This device has been extensively characterized by ESO. Our detector is mounted without a separate detector window; the excellent vacuum performance of our HPF cryostat design ${ }^{8}$ allows us to mount the detector in the same vacuum chamber as the rest of the spectrograph.

Because the camera is mounted near the white pupil of the system in collimated space, the positional tolerances of the camera as a complete subsystem are very loose (on the order of millimeters). Since the power is evenly distributed between the different lens elements and we have avoided steep angles and thin air gaps, the internal alignment tolerances 
of the camera lens elements are moderate as well. We are currently examining the trade-offs between mounting the three main groups of the camera and detector assembly (front triplet, flat fold mirror, singlet plus detector) in separate subassemblies mounted onto the optical bench or integrated inside a common housing.

It is important to note that, although our design was extensively optimized in order to meet the specifications, the local optimum we found is not critically narrow. This fact allows, for example, for straightforward test plate fitting of all radii in the camera with inconsequential degradation of image quality.

\section{CONCLUSION}

In summary, we have developed a single-arm white pupil Echelle spectrograph design for NASA's extreme precision Doppler spectrometer to be installed at the $3.5 \mathrm{~m}$ WIYN telescope. Our design is based on a classic single mirror white pupil relay with unity magnification and a prism cross disperser. The refractive camera has four all-spherical lens elements and provides excellent image quality and high throughput over the entire spectral range (380-930 nm) of the instrument. Our proposal was selected by NASA in April 2016, and we are currently working with vendors to finalize the design and start producing the optics and optomechanics. NEID is scheduled for delivery in 2019.

\section{ACKNOWLEDGEMENTS}

This work was performed in part under contract with the California Institute of Technology (Caltech)/Jet Propulsion Laboratory (JPL) funded by NASA through the Sagan Fellowship Program executed by the NASA Exoplanet Science Institute.

\section{REFERENCES}

[1] Mahadevan, S., Halverson, S., Ramsey, L. and Venditti, N. "Suppression of fiber model noise induced radial velocity errors for bright emission-line calibration sources," The Astrophysical Journal 786, 18-24 (2014).

[2] Baranne, A., "White Pupil Story or Evolution of a Spectrographic Mounting," Very Large Telescopes and their Instrumentation, ESO Conference and Workshop Proceedings 2, 1195-1206 (1988).

[3] Schwab, C., et al., "Adaptive optics fed single-mode spectrograph for high-precision Doppler measurements in the near-infrared," Proc. SPIE 9912, 9912249 (2016).

[4] Stefansson, G. K., et al., "Ultra-stable temperature and pressure control for the Habitable Zone Planet Finder Spectrograph,”, Proc. SPIE 9908, 9908266 (2016); Stefansson, G. K., et al., “A versatile technique to enable sub-milli-kelvin instrument stability for precise radial velocity measurements: tests with the Habitable Zone Planet Finder," in preparation (2016).

[5] Pepe, F., Mayor, M., Delabre, B., Kohler, D., Lacroisx, D., Queloz, D., Udry, S., Benz, W., Betreaux, J.-L. and Sivan, J.-P., "HARPS: a new high-resolution spectrograph for the search of extrasolar planets" Proc. SPIE 4008, 582-592 (2000).

[6] Kaufer, A. and Pasquini, L. "FEROS: the new fiber-linked echelle spectrograph for the ESO 1.52-m telescope," Proc. SPIE 3355, 844-854 (1998).

[7] Andrew Phillips, priv. comm. (2015).

[8] Robertson, P. M., Hearty, F. R., Stefánsson, G. K., Levi, E. I., Bender, C. F., Mahadevan, S., Halverson, S. P., Monson, A. J., Ramseym L. W., Roy, A., Schwab, C., Terrien, R. C., Nelson, M. J. and Blank, B., "A system to provide sub-milliKelvin temperature control at T 300K for extreme precision optical radial velocimetry," Proc. SPIE 9908, 9908228 (2016). 